\title{
Immunogenomic Profiling and Pathological Response Results From a Clinical Trial of Docetaxel and Carboplatin in Triple Negative Breast Cancer
}

Foluso 0. Ademuyiwa ( $\sim$ bisiademuyiwa@wustl.edu )

Washington University School of Medicine https://orcid.org/0000-0002-6766-2258

Ina Chen

Washington University School of Medicine

Jingqin Luo

Washington University School of Medicine

Mothaffar F. Rimawi

Baylor College of Medicine

lan S. Hagemann

Washington University School of Medicine

\section{Bryan Fisk}

Washington University School of Medicine

\section{Gejae Jeffers}

Washington University School of Medicine

Zachary L. Skidmore

Washington University School of Medicine

Anamika Basu

Washington University School of Medicine

Megan Richters

Washington University School of Medicine

Cynthia X. Ma

Washington University School of Medicine

Katherine Weilbaecher

Washington University School of Medicine

Jennifer Davis

Washington University School of Medicine

Rama Suresh

Washington University School of Medicine

Lindsay L. Peterson

Washington University School of Medicine

Ron Bose 
Washington University School of Medicine

\section{Nusayba Bagegni}

Washington University School of Medicine

\section{Caron E. Rigden}

Washington University School of Medicine

\section{Ashley Frith}

Washington University School of Medicine

Timothy P. Rearden

Washington University School of Medicine

\section{Leonel Hernandez-Aya}

Washington University School of Medicine

\section{Anna Roshal}

Washington University School of Medicine

\section{Katherine Clifton}

Washington University School of Medicine

Mateusz Opyrchal

Washington University School of Medicine

Olaronke Akintola-Ogunremi

Christian Hospital NE/NW

\section{Byung Ha Lee}

NeolmmuneTech

\section{Sara Ferrando-Martinez}

NeolmmuneTech

\section{Sarah E. Church}

NanoString Technologies

\section{Meenakshi Anurag}

Baylor College of Medicine

\section{Matthew J. Ellis}

Baylor College of Medicine

\section{Feng Gao}

Washington University School of Medicine

\section{William Gillanders}

Washington University School of Medicine

\section{Obi L. Griffith}

Washington University School of Medicine

\section{Malachi Griffith}

Washington University School of Medicine 


\section{Research article}

Keywords: Breast cancer, clinical trials, combination chemotherapy, immune biomarkers, genomic biomarkers.

Posted Date: May 4th, 2021

DOI: https://doi.org/10.21203/rs.3.rs-420931/v1

License: (c) (i) This work is licensed under a Creative Commons Attribution 4.0 International License. Read Full License 


\section{Abstract}

Purpose: Patients with triple negative breast cancer (TNBC) who do not achieve pathological complete response $(\mathrm{pCR})$ following neoadjuvant chemotherapy have a high risk of recurrence and death. Molecular characterization may identify patients unlikely to achieve $\mathrm{PCR}$. This neoadjuvant trial was conducted to determine the $\mathrm{PCR}$ rate with docetaxel and carboplatin, and to identify molecular alterations and/or immune gene signatures predicting $\mathrm{PCR}$.

Experimental Design: Patients with clinical stages II/III TNBC received 6 cycles of docetaxel and carboplatin. The primary objective was to determine if neoadjuvant docetaxel and carboplatin would increase the pCR rate in TNBC compared to historical expectations. We performed whole exome sequencing (WES) and immune profiling on pre-treatment tumor samples to identify alterations that may predict $\mathrm{pCR}$. Thirteen matching on-treatment samples were also analyzed to assess changes in molecular profiles.

Results: Fifty-eight of 127 (45.7\%) patients achieved pCR. There was a non-significant trend towards higher mutation burden for patients with residual cancer burden (RCB) $0 /$ I versus RCB II/III (median 80, versus 68 variants, $p 0.88)$. TP53 was the most frequently mutated gene, observed in $85.7 \%$ of tumors. EGFR, RB1, RAD51AP2, SDK2, L1CAM, KPRP, PCDHA1, CACNA1S, CFAP58, COL22A1, and COL4A5 mutations were observed almost exclusively in pre-treatment samples from patients who achieved pCR. Seven mutations in PCDHA1 were observed in pre-treatment samples from patients who did not achieve pCR. Several immune gene signatures including ID01, PD-L1, interferon gamma signaling, CTLA4, cytotoxicity, tumor inflammation signature, inflammatory chemokines, cytotoxic cells, lymphoid, PD-L2, exhausted CD8, Tregs, and immunoproteosome were upregulated in pre-treatment samples from patients who achieved pCR.

Conclusions: Neoadjuvant docetaxel and carboplatin resulted in a pCR of $45.7 \%$. WES and immune profiling differentiated patients with and without PCR.

Trial registration: Clinical trial information: NCT02124902, Registered 24 April 2014, \& NCT02547987, Registered 10 September 2015.

\section{Introduction}

Triple negative breast cancer (TNBC) is a heterogeneous clinical breast cancer subtype characterized by the absence of expression of receptors for estrogen (ER), progesterone (PR), and the lack of overexpression of the tyrosine kinase cell surface receptor HER2/ Neu (HER2). This disease subset accounts for approximately $15-20 \%$ of all patients with primary breast cancer.[1] TNBC is characterized by a poorer prognosis compared to other clinical subtypes, and because of the absence of targetable receptors, chemotherapy remains the principal systemic therapy. 
Neoadjuvant chemotherapy is widely used in early stage patients with TNBC who are being treated with curative intent. The preoperative approach provides an opportunity to assess in vivo responses, enables rapid identification of effective drugs, and allows tailoring of adjuvant systemic therapy.[2] Due to improvements in mortality, anthracycline and taxane-based regimens are widely used in the neoadjuvant treatment of patients with TNBC.[3] Unfortunately, anthracyclines are associated with increased cardiac mortality, myelodysplastic syndromes, and treatment-related leukemia. $[3,4]$ Thus, there is interest in evaluating other effective regimens for patients with TNBC. Platinum salts induce double-strand DNA damage and are active in BRCA-associated breast cancers. [5, 6] Sporadic TNBC and BRCA-associated breast cancers share similar molecular features,[7] suggesting possible benefits of platinum salts in patients with TNBC. Recent studies have shown that platinum plus taxane-based non-anthracycline regimens may be an alternative in TNBC patients.[8-11]

The failure of chemotherapy to eradicate disease is believed to be due to selection of cells intrinsically resistant to chemotherapy.[12] Only 30-50\% of patients with TNBC who receive neoadjuvant chemotherapy achieve a pathological complete response (pCR).[13-16] Patients who do not achieve a PCR tend to have a higher rate of recurrence and poorer overall survival than patients who do achieve pCR.[17-20] The 3-year risk of distant recurrence for non-pCR patients is $27 \%$ versus $9 \%$ for those achieving pCR.[21] Three year survival probability is only $68 \%$ in non-pCR versus $94 \%$ in TNBC patients who achieve pCR.[17] The median survival once TNBC has recurred is only 13-25 months.[22-24]

There is no reliable method for predicting which individual patient will achieve $\mathrm{pCR}$, consequently, many early stage patients with TNBC may be exposed to several months of ineffective chemotherapy prior to definitive breast surgery. The ability to individualize chemotherapy is yet to be achieved because robust predictive markers for chemotherapy response have not been identified. We therefore sought to determine the PCR rate with a non-anthracycline regimen of docetaxel and carboplatin in TNBC. Using this clinical trial as a platform for biomarker discovery, we also sought to understand the role of the host and tumor immune profile and tumor genomics in pathological responses.

\section{Materials And Methods Patient population}

Eligible patients included pre- or post-menopausal women at least 18 years old, with clinical stages II or III ER negative (Allred score $<3$ or less than $1 \%$ positive staining cells in the invasive component of the tumor) and HER2 negative ( 0 or $1+$ by IHC or FISH negative) invasive breast cancer. Additional eligibility criteria include: Eastern Cooperative Oncology Group Performance Status of 0 to 2, adequate organ and marrow function, tumor size $\geq 2 \mathrm{~cm}$ in one dimension by clinical or radiographic exam (World Health Organization criteria), and patients with palpable lymph nodes regardless of tumor size. Exclusion criteria included prior treatment of the current cancer, uncontrolled intercurrent illness, bilateral or inflammatory cancer, pregnant/nursing, and prior sentinel lymph node biopsy. The study was approved by the Institutional Review Boards at the participating sites (Washington University School of Medicine [WUSM] 
and Baylor College of Medicine [BCM]), and followed the Declaration of Helsinki and Good Clinical Practice guidelines. Written informed consent was required. Both institutions ran parallel protocols. Clinical trial information: NCT02124902 \& NCT02547987.

\section{Study procedures}

All patients were treated with neoadjuvant intravenous docetaxel $75 \mathrm{mg} / \mathrm{m}^{2}$ and carboplatin AUC 6 cycled every 21 days for 6 cycles, with granulocyte colony-stimulating factor support. Dose adjustments for toxicity were at the discretion of the treating physician. Definitive surgery was performed 3-5 weeks after completion of chemotherapy. Patients received adjuvant radiation when indicated, and adjuvant chemotherapy for patients without PCR was left to the discretion of the treating physician. NCI CTCAE 4.0 was used to record severity and attribution of toxicities. Research tumor biopsies for correlative studies were obtained at baseline prior to chemotherapy, on cycle 1 day 3 (C1D3), and at time of definitive surgery following neoadjuvant chemotherapy in those patients with residual disease. On-treatment biopsy on C1D3 and biopsy at time of relapse were optional. Figure 1 shows the trial schema.

\section{Residual cancer burden (RCB) scoring}

Histologic slides from surgical cases post neoadjuvant therapy were reviewed by a breast pathologist $(\mathrm{IH})$, to determine tumor bed size, percent neoplastic cellularity within the tumor bed, percent of residual tumor that was in situ, number of positive nodes, and largest lymph node deposit. RCB score and category were assigned by the MD Anderson method using a publicly available web calculator (http://www3.mdanderson.org/app/medcalc/index.cfm?pagename=jsconvert3, accessed 9/9/20).

\section{Tumor infiltrating lymphocytes (TILs) assessment}

TILs were quantitated according to the method recommended by the International Immuno-Oncology Biomarker Working Group on Breast Cancer.[25] A breast pathologist $(\mathrm{IH})$ independently reviewed whole slide scans (blinded to treatment status) and documented the percentage of TIL infiltration in increments of $5 \%$ in tumor-adjacent stroma.

\section{Whole exome sequencing (WES)}

Tumor DNA was extracted from fresh-frozen biopsies and matched leukocyte germline DNA from blood samples. WES libraries were enriched using the NimbleGen Roche VCRome v2.1 hybrid capture reagent supplemented with a "panel killer" spike-in designed by BCM. Paired-end ( $2 \times 150$ base pair) next generation sequencing was performed using the Illumina platform to a target depth of coverage of $100 \mathrm{x}$ (mean target coverage achieved was 70-130x across the cohort). Sequence data processing pipelines utilized tools implemented in Docker containers (published to DockerHub), input parameters and data specified in YAML configuration files, pipeline steps and dependencies expressed using the Common Workflow Language (CWL), and compute tasks submitted to a compute cluster using Cromwell and platform LSF.[26] All associated CWL files, example YAML files, and docker files are version tracked using Git and GitHub (https://github.com/genome/analysis-workflows). Metadata on analysis runs was tracked using an Analysis Information Management System developed at the Washington University McDonnell 
Genome Institute.[27] Briefly, WES analysis was performed by aligning sequence reads to the human reference genome build GRCh38 using the BWA-MEM aligner.[28] Alignments were subjected to base quality score recalibration,[29] sorted by chromosome position, duplicates marked with Picard (http://broadinstitute.github.io/picard/), and converted to a lossless CRAM format (https://github.com/samtools/hts-specs) to reduce disk usage. All samples in each cohort were tested for sample swaps and contamination using Somalier (https://github.com/brentp/somalier) and subjected to a QC analysis consisting of Picard, samtools flagstat,[30] and VerifyBamID (https://github.com/statgen/verifyBamID). Somatic single nucleotide variants (SNVs) and small insertions and deletions were called using an ensemble approach involving MuTect2, Strelka2, Varscan2 and Pindel.[31-33] Somatic variants from each of these callers were left aligned and trimmed using GATK LeftAlignAndTrim,[29] merged into a single variant call format file using GATK CombineVariants and multiallelic sites separated using vt decompose.[34] Somatic variants were subjected to a false positive filter to flag variants of high frequency in the population according to GNOMAD,[35] lacking minimum sequence read support from both sequence strands, corresponding to regions of the genome with ambiguous read mapping, and those that fail a log likelihood test that models the observed read support relative to known error rates of the sequencing platform and conservative sample specific assumptions of tumor purity. The resulting candidate somatic variants were subjected to a formal manual review standard operating procedure.[36] The resulting high confidence somatic variants were annotated for transcript variant effect using VEP and Ensembl transcripts.[37, 38] Variant allele frequencies were computed using bam-readcount (https://github.com/genome/bam-readcount). Pathway analyses for genes harboring sub-clonal variants selected under treatment pressure were performed using WEB-based GEne SeT AnaLysis Toolkit [39] using an Over-Representation Analysis (ORA) approach and "Wikipathway cancer" as the source of pathways.

\section{NanoString gene expression analysis}

RNA extracted from formalin-fixed paraffin embedded (FFPE) tissue samples were analyzed on the nCounter ${ }^{\circledR}$ analysis system using the PanCancer $10360^{\mathrm{Tm}}$ panel (for research use only). Raw data counts were normalized using the geomean of 20 housekeeping genes in the I0360 panel and each gene was adjusted based on 10360 panel standards to adjust for batch-to-batch variation. The housekeepernormalized and panel standard-normalized data is Log(2) transformed. 10360 gene analysis for 48 signatures measuring immune cell abundance, immune signaling, tumor and stromal biology were calculated as previously described.[40,41] A constant of 8 is added to the tumor inflammation signature (TIS) so that it is on the same scale as investigational use only (IUO) TIS, making scores comparable across research use only (RUO) and IUO assays. Other non-TIS signatures are also adjusted with constants to express values in a similar range.

\section{Statistical analyses}

The primary endpoint of the clinical trial is $\mathrm{PCR}$ rate calculated as the percentage of patients who achieve pCR among all evaluable patients. pCR is defined as absence of residual invasive disease in the breast and lymph nodes following neoadjuvant chemotherapy. Exploratory aims were to investigate immune 
and genomic changes with the intent to identify predictors of response. A sample size of 100 patients provides $82.1 \%$ power to test a pCR rate of $40 \%$ against the null rate of $28 \%$ with standard chemotherapy, based on 1-sided binomial exact test at a target 0.05 alpha level. If 36 or more patients achieve a pCR, we conclude that the investigational regimen yields better efficacy than standard chemotherapy.

Patient characteristics were summarized by descriptive statistics, counts and percentages for categorical characteristics, and median with inter-quartile ranges (IQR) for quantitative characteristics. Tumor burden was compared between PCR (RCB 0) versus non-pCR patients, and between RCB 0/I versus RCB II/III patients, by Wilcoxon rank sum test, and compared between paired pre-treatment and C1D3 samples by Wilcoxon signed rank test. Mutation landscape waterfall plot was generated using the R package "GenVisR" (Version 1.16.1). Gene sets defined in the Molecular Signatures Database v7.1 (MSigDB v7.1 released March 2020) were downloaded and extracted using R package "msigdbr". The overall effect of gene mutations of a gene set on pathological outcome was evaluated using the sequence kernel based association (SKAT) test method in the logistic regression framework. SKAT $p$ value was reported for each gene set.

NanoString IO360 signatures were compared based on pathological response and time point. Differential expression based on response was fit on a per gene or per signature basis using a linear model for analyses without a blocking factor. The statistical model uses the expression value or signature score as the dependent variable and fits a grouping variable as a fixed effect to test for differences in the levels of that grouping variable.

Expression (gene or signature) $=\mu+$ Response $+\varepsilon$

For differential expression for time series analysis the duplicateCorrelation function within the limma $\mathrm{R}$ package is used to assess the correlation between subsequent time points. This correlation estimate is fit into the linear mixed effect model with subject as the random effect and the correlation between the repeated temporal measurements.

Expression $_{- \text {(gene or signature) }}=\mu+$ SubjectID + Group $+\varepsilon$

For all differential expression analysis. All models are fit using the limma package in R.

\section{Results}

\section{Patient characteristics and clinical efficacy}

Between $8 / 2014$ and 1/2020, 168 patients were screened and 132 ultimately received protocol therapy. Thirty-five did not meet inclusion criteria/were not registered due to reasons such as being found to have estrogen receptor positive breast cancer, metastatic disease, or abnormal laboratory values. Five patients were not evaluable for $\mathrm{pCR}$ due to a variety of reasons, including patient or physicians choice to withdraw protocol therapy. One hundred and twenty seven patients were evaluable. Median follow up is 27 months 
(IQR 14.4-39.5 months). Additional file 1 shows the patient disposition. Median age at diagnosis was 52.5 years (IQR 42-61). African Americans comprised $23.5 \%$ of the study population, $93.2 \%$ had high grade disease, and $43.9 \%$ had clinical nodal involvement prior to chemotherapy. Patient characteristics are shown in Table 1.

Table 1. Characteristics of study population

\begin{tabular}{|c|c|c|c|c|}
\hline & $\begin{array}{l}\text { Total study population } \\
\text { (\%) }\end{array}$ & $\begin{array}{l}\text { Non-pCR N=69 } \\
(\%)\end{array}$ & pCR N=58 (\%) & $\begin{array}{l}p \\
\text { value }\end{array}$ \\
\hline \multicolumn{5}{|c|}{$\begin{array}{l}\text { Age at diagnosis, median } \\
\text { (IQR) }\end{array}$} \\
\hline & $52.5(42-61)$ & $53.0(44.0-62.0)$ & $\begin{array}{l}51.5(40.2- \\
58.8)\end{array}$ & 0.301 \\
\hline \multicolumn{5}{|l|}{ Race } \\
\hline Caucasian & $98(74.2)$ & $49(71.0)$ & $44(75.9)$ & \\
\hline Black & $31(23.5)$ & $18(26.1)$ & $13(22.4)$ & \\
\hline Other & $3(2.3)$ & $2(2.9)$ & $1(1.7)$ & 0.913 \\
\hline \multicolumn{5}{|c|}{ Clinical stage } \\
\hline ॥ & $107(81.1)$ & $54(78.3)$ & $48(82.8)$ & \\
\hline III & $25(18.9)$ & $15(21.7)$ & $10(17.2)$ & 0.648 \\
\hline \multicolumn{5}{|c|}{ Tumor grade } \\
\hline ॥ & $9(6.8)$ & $7(10.1)$ & $2(3.5)$ & \\
\hline III & $123(93.2)$ & $62(89.9)$ & $56(96.5)$ & 0.179 \\
\hline \multicolumn{5}{|c|}{ Clinical node status } \\
\hline Positive & $58(43.9)$ & $34(49.3)$ & $22(37.9)$ & \\
\hline Negative & $74(56.1)$ & $35(50.7)$ & $36(62.1)$ & 0.301 \\
\hline
\end{tabular}

Fifty-eight of 127 (45.7\%) evaluable patients achieved pCR (95\% Cl 36.9\%-54.7\%), and pCR was similar by accrual site (WUSM: 45.6\%, 95\% Cl 35.9\% - 55.7\% and BCM: 45.8\%, 95\% Cl 26.2\% - 66.8\%). There were no differences in age, race, clinical stage, grade, or clinical node status according to pathological response (Table 1 ).

\section{Toxicity}

All patients who received at least 1 cycle of combination chemotherapy are evaluable for toxicity. Treatment-emergent adverse events of any grade occurred in 123 patients, with 1,947 events reported. Table 2 shows the incidence of grade 3 and 4 adverse events occurring in at least two patients. The incidence of grade 3 to 4 anemia was $18.2 \%$, thrombocytopenia was $13.6 \%$, diarrhea $9.1 \%$, and febrile 
neutropenia $7.6 \%$. Adverse events leading to discontinuation of the regimen were reported in 15 patients $(11.4 \%)$. Nineteen patients (14.4\%) had at least one SAE. (Table 2). No treatment-related deaths occurred. 
Table 2

Treatment-emergent adverse events of $\geq$ grade 3 in study population

All patients, $\mathbf{N}=132$

$\mathbf{N}(\%)$

Total number of grade $\geq 3$ TEAEs occurring in $\geq 2$ patients $56(42.4)$

Anemia

24 (18.2)

Thrombocytopenia

18 (13.6)

Lymphopenia

14 (10.6)

Diarrhea

$12(9.1)$

Febrile neutropenia

10 (7.6)

Neutropenia

10 (7.6)

Hyponatremia

7 (5.3)

Leucopenia

6 (4.5)

Hypokalemia

5 (3.8)

Fatigue

2 (1.5)

Oral mucositis

2 (1.5)

Anorexia

2 (1.5)

Syncope

$2(1.5)$

Renal disorders

$2(1.5)$

Patients with any SAEs

19 (14.4)

SAEs occurring in $\geq 2$ patients

Syncope

Febrile neutropenia

7 (5.3)

Colitis

4 (3.0)

Nausea

$2(1.5)$

Neutropenia

$3(2.3)$

Urinary tract infections

$3(2.3)$

Diarrhea

Acute kidney injury

$2(1.5)$ 
We evaluated the association of immune parameters with pathological response. Stromal TILs, CD4, CD8, CD79a, and PD-L1 levels from tumor samples were assessed by hematoxylin and eosin (H\&E)-stained tumor sections and immunohistochemistry. Higher baseline stromal TILs (OR 1.31; 95\% Cl: 1.00-1.70, p $=0.05)$ and $\operatorname{CD} 8(\mathrm{OR} 1.14 ; 95 \% \mathrm{Cl}: 1.03-1.26, \mathrm{p}=0.02)$ values were associated with $\mathrm{pCR}$.

Patients with higher post-treatment absolute lymphocyte counts (ALCs) from peripheral blood were more likely to achieve pCR than those with lower ALCs (OR 5.5; 95\% Cl: 1.5-20.7, p = 0.011). Similarly, patients with higher minimum ALCs were also more likely to achieve pCR than those with a lower ALC nadir (OR 9.1, 95\% $\mathrm{Cl} 1.5-54.9, \mathrm{p}=0.016)$. The associations of post-treatment and minimum ALCs with $\mathrm{pCR}$ remained significant after adjusting for age and clinical stage at diagnosis (post-treatment ALC OR 7.6; $95 \% \mathrm{Cl}: 1.7-34.8, \mathrm{p}=0.009$; minimum ALC OR 9.0; $95 \% \mathrm{Cl}: 1.5-55.2, \mathrm{p}=0.018)$. Baseline ALC values were not associated with pCR (OR 1.0; 95\% Cl: 0.6-1.9, p = 0.863).

Analysis of gene expression in samples collected at baseline using the NanoString's nCounter® PanCancer IO 360 Gene Expression Panel, demonstrated that several immune signatures, including ID01 $(p=0.004)$, PD-L1 $(p=0.001)$, interferon gamma signaling $(p=0.016)$, CTLA4 $(p=0.038)$, cytotoxicity $(p=$ $0.019)$, TIS $(p=0.026)$, inflammatory chemokines $(p=0.013)$, cytotoxic cells $(p=0.028)$, lymphoid $(p=$ $0.040)$, PD-L2 ( $p=0.018)$, exhausted CD8 ( $p=0.042)$, Tregs $(p=0.035)$, and immunoproteosome $(p=$ 0.028) were upregulated in pCR versus non-pCR samples (Fig. 2). Key immune signatures of borderline significance included T cells, CD8 T cells, IL7R, and TIGIT. A heatmap displayed as Additional file 2 uses unsupervised hierarchical clustering to show relatedness among signature scores for baseline samples according to pathological response.

In a longitudinal analyses, differences between pre- and on-treatment (C1D3) paired samples were analyzed. Many immune-related signatures were significantly higher in baseline samples compared to ontreatment (Fig. 3 and Additional file 3). The signatures with the greatest significance covered four categories of immune biology, including interferon-related (TIS: $p<0.001$, Interferon gamma signaling: $p$ $<0.001$, Interferon signaling: $p<0.005)$, T cell abundance (Exhausted CD8: $p<0.001$, T cells: $p<0.0005$, CD8 T cells: $p<0.005$, Th1 T cells: $p<0.005$ ), cytotoxicity (Cytotoxicity: $p<0.001$, Cytotoxic Cells: $p<$ 0.001) and checkpoint molecules (PD-L2: $p<0.001$, TIGIT: $p<0.001$, PD-L1: $p<0.001$, CTLA4: $p<0.001$, PD-1: $p<0.005)$. Interestingly, baseline $p C R$ samples had slightly higher expression of ID01, inflammatory chemokines, and CD56dim cell abundance signatures at compared to non-pCR samples, and showed a larger change in signatures scores from baseline to on-treatment time points.

\section{Tumor mutation profiling and pathological response}

WES was performed on baseline pre-treatment samples collected from a subset ( $N=56$ patients) of the clinical trial population. Thirteen patients had baseline-matched samples from C1D3 that were analyzed. 9063 variants were detected in 5386 unique genes. The mutation landscape waterfall plot of genes with a mutation frequency of $>5 \%$ is shown in Fig. 4 . The overall mutation burden for patients who achieved pCR was not significantly different from non-pCR patients (median of 78.5 variants, IQR 43-134 in pCR, vs median 72, IQR 47.8-103.8 in non-pCR, Wilcoxon rank sum test $p=0.98$ ). Similarly, there was no 
difference in the overall mutation burden for patients with RCB 0/I versus those with RCB II/III (median of 80 variants, IQR 40-134 in RCB 0/I, vs median 68, IQR 53.5-87.8 in RCB II/III, Wilcoxon rank sum test $p=$ 0.88) (Fig. 5).

Table 3 shows the genes with variants occurring in at least $10 \%$ of the biomarker population, according to pathological response. TP53 was the most frequently mutated gene observed in 48 of 56 patients sequenced (85.7\%) As expected for TP53, we observed mutations primarily within the DNA-binding domain, and many of the mutations are likely loss-of-function variants caused by frameshift insertion/deletions, frameshift splice variants, and nonsense mutations (Additional file 4). There was a non-significant trend with fewer TP53 mutations occurring in $80 \%$ of patients with $\mathrm{pCR}$, versus $88.9 \%$ of non-pCR patients (OR 0.51, 95\% $\mathrm{Cl} 0.08-3.09 ; \mathrm{p}$ value 0.44 ). There were no differences in TP53 mutation frequency in patients with RCB 0/I (85\%), versus RCB II/III (87.5\%) (OR 1.23, 95\% Cl 0.19-13.92; p value 1.00). EGFR, RB1, RAD51AP2, SDK2, L1CAM, KPRP, PCDHA1, CACNA1S, CFAP58, COL22A1, and COL4A5 were differentially mutated, and almost exclusively found in pre-treatment pCR samples (except PCDHA1). The seven mutations in PCDHA1 were observed in non-pCR samples only (19.4\%). We observed four variants within EGFR in three patients. Three of these variants occurred within the tyrosine kinase catalytic domain region, and all three have documented clinical significance with respect to targeted therapy response in CIViC (Additional file 5). One of the patients with an EGFR mutation has recurred, despite $\mathrm{PCR}$.

Table 3

Genes with variants occurring in at least $10 \%$ of the biomarker study population.

\begin{tabular}{|lllll|}
\hline Gene symbol & Variant/N (\%) & Non-pCR N = 36 (\%) & pCR N = 20 (\%) & p value \\
\hline TP53 & $48(85.7)$ & $32(88.7)$ & $16(80.0)$ & 0.44 \\
\hline TTN & $13(23.2)$ & $9(25.0)$ & $4(20.0)$ & 0.75 \\
\hline FLG & $8(14.3)$ & $6(16.7)$ & $2(10.0)$ & 0.69 \\
\hline PCDHA1 & $7(12.5)$ & $7(19.4)$ & $0(0.0)$ & 0.04 \\
\hline TRMT9B & $6(10.7)$ & $5(13.9)$ & $1(5.0)$ & 0.40 \\
\hline PCDHGA2 & $6(10.7)$ & $3(8.3)$ & $3(15.0)$ & 0.66 \\
\hline FAM135B & $6(10.7)$ & $4(11.1)$ & $2(10.0)$ & 1.00 \\
\hline KIAA1671 & $6(10.7)$ & $4(11.1)$ & $2(10.0)$ & 1.00 \\
\hline SPEG & $6(10.7)$ & $4(11.1)$ & $2(10.0)$ & 1.00 \\
\hline TENM1 & $6(10.7)$ & $4(11.1)$ & $2(10.0)$ & 1.00 \\
\hline
\end{tabular}

In the longitudinal analyses, there was evidence of tumor heterogeneity and shifts in clonal architecture under treatment pressure from pre-treatment to C1D3, due to both selection and depletion of subclones. However, more variants were enriched suggesting possible subclone emergence under treatment pressure (Fig. 6). The overall variant counts in the matched samples at C1D3 trended higher (median of 82, IQR 
49-157) than corresponding pre-treatment samples (median of 72, IQR 42-92), $p=0.29$. As expected, all tumor pairs had a substantial set of shared clonal variants with TP53 variants exhibiting high variant allele frequency (VAF) reflecting its driver status. Across all patients, 289 genes harbored such emerging variants including a second TP53 Y220C variant in patient NTN022 (0\% VAF at baseline and $13.0 \%$ VAF at C1D3), and an EGFR S768I variant in NTN046 (0.07\% VAF at baseline and 29.5\% VAF at C1D3). Pathway analysis of the entire set of mutated genes emerging at C1D3 (green points in Fig. 6) showed significant enrichment for DNA damage and nucleotide synthesis pathways suggesting possible selection for cells with molecular mechanisms of resistance to the DNA damaging agent carboplatin.

Last, we explored whether mutations in different gene families would cluster in recognized pathways. Using the Molecular Signatures Database (MsigDB) v7.1, several gene families involved in immunesignature related gene sets (MsigDB C7 set) showed differences between RCB 0/I and RCB II/III samples (Additional file 6). Additionally, borderline differences in PI3K AKT MTOR signaling pathway among MsigDB 50 hallmark gene sets were identified between RCB 0/I and RCB II/III samples. There were no differences in inflammatory response, angiogenesis, apoptosis, NOTCH signaling, TNF alpha, or androgen response pathways.

\section{Discussion}

In this multicenter single-arm phase II trial of neoadjuvant docetaxel and carboplatin in patients with newly diagnosed clinical stage II to III TNBC, we observed a pCR rate of $45.7 \%$. These data are consistent with previous observations that non-anthracycline based regimens in TNBC patients achieve similar pCR rates as anthracycline plus taxane based regimens.[8-11] Recent randomized trials demonstrate that the addition of carboplatin to anthracycline taxane based regimens increase pCR rates, but also increase toxicity.[13,14] More recently, Sharma et al. compared neoadjuvant carboplatin with docetaxel to carboplatin with paclitaxel followed by anthracyclines in patients with early stage TNBC.[42] This study demonstrated similar pCR rates in both study groups: carboplatin with docetaxel group pCR $52 \%$, versus anthracycline group $\mathrm{pCR} 55 \%, \mathrm{p}=0.84$. Patients who did not receive anthracyclines had a more favorable toxicity profile and higher treatment completion rate compared with patients who received anthracyclines. The effects of the addition of carboplatin to anthracycline based regimens on longer-term clinical outcomes such as event-free and overall survival are conflicting.[43] GeparSixto, a randomized phase 3 trial that evaluated the addition of carboplatin to anthracycline and taxanes in TNBC, showed a higher 3year disease-free survival rate in the carboplatin group vs the non-carboplatin group ( $85 \%$ vs $76 \%, p=$ 0.03). $[14,44]$ Conversely, Cancer and Leukemia Group B (CALGB) 40603 randomized phase II trial demonstrated no difference in 3-year event-free survival with the addition of carboplatin to anthracycline taxane based neoadjuvant therapy $(71 \%$ versus $76 \%, p=0.36)$.[45] As a result, carboplatin has not been incorporated into the routine clinical management of early stage TNBC. Ongoing clinical trials may address long-term outcomes in the future.[46-48] The similarity in antitumor activity with docetaxel and carboplatin compared with standard anthracycline taxane based regimens, and the increased toxicities with adding carboplatin to anthracycline taxane based regimens provide rationale for considering docetaxel and carboplatin chemotherapy for patients with early stage TNBC. Moreover, the long-term risk 
of cardiotoxicity, myelodysplasia, and therapy-associated leukemia is minimized with non-anthracycline regimens.

PCR is a surrogate of long-term outcomes in patients with TNBC, and the extent of residual disease is linked to the risk of recurrence.[16, 17, 49-51] Individuals who do not achieve pCR or RCB I have a high risk of recurrent disease and subsequent death, with a hazard ratio for an overall survival event reported to be as high as $12.4(95 \% \mathrm{Cl} 5.8-26.5, \mathrm{p}=0.001)$. [16, 17] Other than $B R C A$ germline status, $[44,52,53]$ there are no predictive factors for $\mathrm{pCR}$. There are promising data using early imaging changes in positron emission or computed tomography, tumor morphological changes, and ctDNA to predict pathological response to neoadjuvant chemotherapy.[54-57] From a clinical perspective, understanding variables that predict response is an urgent unmet need. If TNBC patients who will not achieve pCR can be identified earlier, they may be triaged to innovative trials or definitive surgery, with a view to changing the natural history of resistant TNBC whilst sparing them the toxicity of ineffective chemotherapy. Therefore, we examined tumor immune and genomic profiles to identify molecular factors that may predict response. Our study did not identify mutation burden as a predictor for pathological response to docetaxel and carboplatin chemotherapy. An interesting observation was the identification of EGFR mutations in $\mathrm{pCR}$ patients. Although EGFR mutations have previously been described in TNBC,[58] the clinical relevance has not been described. These results need to be confirmed in a larger study, but may suggest a potential rationale for evaluating EGFR tyrosine kinase inhibitor therapy in TNBC patients harboring EGFR mutations.

Due to the absence of recurrently mutated genes other than TP53,[59] the aggregation of individual genes at the pathway level may be a more practical way to evaluate predictors of response in TNBC. Ontreatment samples had a suggestion of clone emergence, with increased variants compered to baseline samples. Pathway analysis of the emerging genes showed enrichment for DNA damage pathway, suggesting selection for resistance to the DNA damaging agents. These hypothesis-generating results suggest that early on-treatment tumor assessment may be used in the future to identify patients who may be more likely to respond to chemotherapy. We also found that several immune-related pathway gene signatures showed differences between pathological outcomes, suggesting the possible utility of immune perturbation in early TNBC. Patients with higher baseline TILs, baseline upregulation of immunerelated (IDO, PD-L1, lymphoid, Tregs), inflammation-related (inflammatory cytokines, immunoproteasome) and cytotoxicity-related (interferon gamma signaling, cytotoxic cells, exhausted CD8) gene signatures were more likely to achieve pCR. In addition, patients that were able to maintain higher post-treatment absolute lymphocyte counts despite the lymphodepleting effects of chemotherapy, were also more likely to achieve pCR. Taken together, these results suggest that patients with an inflamed tumor microenvironment (TME) that experience less treatment-related lymphopenia may be more responsive to neoadjuvant chemotherapy with docetaxel and carboplatin. Thus, immunomodulatory interventions such as IL-7 treatment which is aimed at increasing the persistence, survival and trafficking of lymphocytes may provide a significant clinical benefit when combined with neoadjuvant strategies. Long-acting recombinant human IL-7 (rhIL-7-hyFc) has shown to be safe and well tolerated in human, while significantly increasing the frequency of lymphocytes.[60] rhIL-7-hyFc has also proven to increase T cell 
infiltration and inflammation within the TME in cancer-bearing mouse models.[61] Using rhIL-7-hyFc treatment to improve the rates of $\mathrm{pCR}$ in TNBC patients undergoing adjuvant chemotherapy is an appealing strategy that needs further investigation.

Strengths of this translational study include the high proportion of African Americans accrued, which is likely reflective of the demographics of both accrual sites, and the commitment of the investigators to accrue minorities to clinical trials. Furthermore, this was a multicenter collaborative study, which simultaneously served as a platform for biomarker analyses. Additionally, this was a homogenously treated, clinically well-annotated TNBC cohort. All samples were processed similarly at the same institution, the whole exome sequencing approach utilized was comprehensive, and we had normal tissue on all patients for accurate variant calling. However, several limitations deserve comment. First, this is a phase 2 single-arm non-randomized study. Therefore, a larger randomized trial is necessary for further evaluation of non-athracycline based regimens in TNBC. Second, the relatively small sample size of the correlative study limits the power of the analyses. Follow up is short but ongoing, and long-term clinical outcomes will be reported as results become available. Despite these limitations, this is the first hybrid study with comprehensive tumor profiling comparing a homogeneously treated cohort of TNBC patients to identify biomarkers predicting $\mathrm{PCR}$.

\section{Conclusions}

Our results demonstrate a robust $\mathrm{pCR}$ rate of $45.7 \%$ with this alternative non-anthracycline chemotherapy regimen administered to patients with TNBC. Tumor mutation assessment for EGFR, RB1, RAD51AP2, SDK2, L1CAM, KPRP, PCDHA1, CACNA1S, CFAP58, COL22A1, COL4A5 mutations, and immune-related gene signatures may discriminate patients who will achieve $\mathrm{pCR}$ following platinum-based neoadjuvant chemotherapy. While larger confirmatory studies are needed, these may be potential biomarkers for predicting pathological response and therapeutic efficacy in patients with TNBC, and emphasize the need for molecular analyses in therapeutic clinical trials.

\section{Abbreviations}

TNBC Triple negative breast cancer

ER Estrogen receptor

PR Progesterone receptor

HER2 HER2/ Neu gene

DNA Deoxyribonucleic acid

pCR Pathological complete response

IHC Immunohistochemistry 
FISH Fluorescence in situ hybridization

WUSM Washington University School of Medicine

BCM Baylor College of Medicine

AUC Area under the curve

NCI CTCAE National Cancer Institute Common Terminology Criteria for Adverse Events

C1D3 Cycle 1 day 3

RCB Residual cancer burden

TIL Tumor infiltrating lymphocytes

WES Whole exome sequencing

SNV Single nucleotide variant

RNA Ribonucleic acid

FFPE Formalin fixed paraffin embedded

TIS Tumor inflammation signature

IUO Investigational use only

RUO Research use only

SKAT Sequence kernel based association

SAE Serious adverse events

ALC Absolute lymphocyte count

OR Odds ratio

VAF Variant allele frequency

TME Tumor microenvironment

\section{Declarations}

\section{Ethical Approval and Consent to participate}


The protocol and informed consent documents were approved by WUSM and BCM. Upon approval, all participating institutions agreed to follow the Declaration of Helsinki, good clinical practice guidelines, and the applicable parts of the U.S. Code of Federal Regulations. Written informed consent was required for enrollment.

\section{Consent for publication}

Not applicable.

\section{Availability of supporting data}

The datasets used and/or analyzed during the present study are available from the corresponding author on reasonable request.

\section{Competing interests}

FOA reports consulting for Eisai, Immunomedics, Astra Zeneca, Athenex, Cardinal Health, Pfizer, Abbvie, Best Doctors, and Advance Medical. FOA reports contracted research for Immunomedics, Pfizer, Seattle Genetics, NeolmmuneTech, RNA Diagnostics, and Astellas. MFR reports consulting for Genentech, MacroGenics, Daiichi, Seattle Genetics, and Novartis. MFR reports contracted research for Pfizer. RB consulting for Genentech. RB reports contracted research for Puma Biotechnology, Inc.

\section{Funding}

FOA- Research reported in this publication was supported by the National Cancer Institute of the National Institutes of Health under Award Number K12 CA167540. The content is solely the responsibility of the authors and does not necessarily represent the official views of the NIH. Research reported in this publication was supported by NeolmmuneTech, Inc.

MJE- Research reported in this publication was supported by the National Cancer Institute of the National Institutes of Health under Award Number U01 CA214125-03. The content is solely the responsibility of the authors and does not necessarily represent the official views of the NIH. Research reported in this publication was supported by the Cancer Prevention \& Research Institute of Texas Established Investigator Award RR140033. The content is solely the responsibility of the authors and does not necessarily represent the official views of the CPRIT.

OLG- Research reported in this publication was supported by the National Cancer Institute of the National Institutes of Health under Award Number K22CA188163. The content is solely the responsibility of the authors and does not necessarily represent the official views of the $\mathrm{NIH}$.

MG- Research reported in this publication was supported by the National Human Genome Research Institute of the National Institutes of Health under Award Number R0OHG007940. The content is solely the responsibility of the authors and does not necessarily represent the official views of the $\mathrm{NIH}$. 


\section{Authors' contributions}

FOA, IC, MJE, WG were involved in the conception and design of the study. FOA, MFR, CXM, KW, RS, LLP, $\mathrm{RB}, \mathrm{NB}, \mathrm{CER}, \mathrm{AF}, \mathrm{TPR}, \mathrm{LFH}, \mathrm{AR}, \mathrm{KC}, \mathrm{MO}$ participated in the acquisition of data (acquired and managed patients, provided facilities, etc.). FOA, IC, JL, MFR, ISH, BF, GJ, ZLS, AB, MR, CXM, KW, JD, LLP, RB, MO, $\mathrm{OA}, \mathrm{BHL}, \mathrm{SF}, \mathrm{SC}, \mathrm{MA}, \mathrm{FG}, \mathrm{WG}, \mathrm{OLG}, \mathrm{MG}$ provided analysis and interpretation of data (e.g., statistical analysis, biostatistics, computational analysis). All authors were involved in writing, reviewing, and/or revision of the manuscript. FOA, BF, GJ, ZLS, AB, MR, SC, MA, OLG, MG provided administrative, technical, or material support (i.e., reporting or organizing data, constructing databases). The authors read and approved the final manuscript.

\section{Acknowledgments}

Tracy Summa, Isabella Grigsby, Christina Robinson, and Kristen Otte for providing clinical research support.

\section{References}

1. DeSantis CE, Ma J, Gaudet MM, Newman LA, Miller KD, Goding Sauer A, Jemal A, Siegel RL. Breast cancer statistics, 2019. CA Cancer J Clin. 2019;69(6):438-51.

2. Masuda N, Lee SJ, Ohtani S, Im YH, Lee ES, Yokota I, Kuroi K, Im SA, Park BW, Kim SB, et al. Adjuvant Capecitabine for Breast Cancer after Preoperative Chemotherapy. N Engl J Med. 2017;376(22):214759.

3. Early Breast Cancer Trialists'. Collaborative G, Peto R, Davies C, Godwin J, Gray R, Pan HC, Clarke M, Cutter D, Darby S, McGale P, et al: Comparisons between different polychemotherapy regimens for early breast cancer: meta-analyses of long-term outcome among 100,000 women in 123 randomised trials. Lancet 2012, 379(9814):432-44.

4. Henderson IC, Berry DA, Demetri GD, Cirrincione CT, Goldstein LJ, Martino S, Ingle JN, Cooper MR, Hayes DF, Tkaczuk KH, et al. Improved outcomes from adding sequential Paclitaxel but not from escalating Doxorubicin dose in an adjuvant chemotherapy regimen for patients with node-positive primary breast cancer. Journal of clinical oncology: official journal of the American Society of Clinical Oncology. 2003;21(6):976-83.

5. Byrski T, Dent R, Blecharz P, Foszczynska-Kloda M, Gronwald J, Huzarski T, Cybulski C, Marczyk E, Chrzan R, Eisen A, et al. Results of a phase II open-label, non-randomized trial of cisplatin chemotherapy in patients with BRCA1-positive metastatic breast cancer. Breast Cancer Res. 2012;14(4):R110.

6. Byrski T, Huzarski T, Dent R, Gronwald J, Zuziak D, Cybulski C, Kladny J, Gorski B, Lubinski J, Narod SA. Response to neoadjuvant therapy with cisplatin in BRCA1-positive breast cancer patients. Breast Cancer Res Treat. 2009;115(2):359-63. 
7. Sorlie T, Tibshirani R, Parker J, Hastie T, Marron JS, Nobel A, Deng S, Johnsen H, Pesich R, Geisler S, et al. Repeated observation of breast tumor subtypes in independent gene expression data sets. Proc Natl Acad Sci U S A. 2003;100(14):8418-23.

8. Kern P, Kalisch A, Kolberg HC, Kimmig R, Otterbach F, von Minckwitz G, Sikov WM, Pott D, Kurbacher C. Neoadjuvant, anthracycline-free chemotherapy with carboplatin and docetaxel in triple-negative, early-stage breast cancer: a multicentric analysis of feasibility and rates of pathologic complete response. Chemotherapy. 2013;59(5):387-94.

9. Kern P, Kalisch A, von Minckwitz G, Putter C, Kolberg HC, Pott D, Kurbacher C, Rezai M, Kimmig R. Neoadjuvant, anthracycline-free chemotherapy with carboplatin and docetaxel in triple-negative, early-stage breast cancer: a multicentric analysis of rates of pathologic complete response and survival. J Chemother. 2016;28(3):210-7.

10. Sharma P, Lopez-Tarruella S, Garcia-Saenz JA, Khan QJ, Gomez HL, Prat A, Moreno F, Jerez-Gilarranz Y, Barnadas A, Picornell AC, et al. Pathological Response and Survival in Triple-Negative Breast Cancer Following Neoadjuvant Carboplatin plus Docetaxel. Clinical cancer research: an official journal of the American Association for Cancer Research. 2018;24(23):5820-9.

11. Sharma P, Lopez-Tarruella S, Garcia-Saenz JA, Ward C, Connor CS, Gomez HL, Prat A, Moreno F, Jerez-Gilarranz Y, Barnadas A, et al. Efficacy of Neoadjuvant Carboplatin plus Docetaxel in TripleNegative Breast Cancer: Combined Analysis of Two Cohorts. Clinical cancer research: an official journal of the American Association for Cancer Research. 2017;23(3):649-57.

12. Aparicio $S$, Caldas $C$. The implications of clonal genome evolution for cancer medicine. $N$ Engl $J$ Med. 2013;368(9):842-51.

13. Sikov WM, Berry DA, Perou CM, Singh B, Cirrincione CT, Tolaney SM, Kuzma CS, Pluard TJ, Somlo G, Port ER, et al. Impact of the addition of carboplatin and/or bevacizumab to neoadjuvant once-perweek paclitaxel followed by dose-dense doxorubicin and cyclophosphamide on pathologic complete response rates in stage II to III triple-negative breast cancer: CALGB 40603 (Alliance). Journal of clinical oncology: official journal of the American Society of Clinical Oncology. 2015;33(1):13-21.

14. von Minckwitz G, Schneeweiss A, Loibl S, Salat C, Denkert C, Rezai M, Blohmer JU, Jackisch C, Paepke S, Gerber B, et al. Neoadjuvant carboplatin in patients with triple-negative and HER2-positive early breast cancer (GeparSixto; GBG 66): a randomised phase 2 trial. Lancet Oncol. 2014;15(7):747-56.

15. Gerber B, LoibI S, Eidtmann H, Rezai M, Fasching PA, Tesch H, Eggemann H, Schrader I, Kittel K, Hanusch $\mathrm{C}$, et al. Neoadjuvant bevacizumab and anthracycline-taxane-based chemotherapy in 678 triple-negative primary breast cancers; results from the geparquinto study (GBG 44). Ann Oncol. 2013;24(12):2978-84.

16. von Minckwitz G, Untch M, Blohmer JU, Costa SD, Eidtmann H, Fasching PA, Gerber B, Eiermann W, Hilfrich $\mathrm{J}$, Huober $\mathrm{J}$, et al. Definition and impact of pathologic complete response on prognosis after neoadjuvant chemotherapy in various intrinsic breast cancer subtypes. Journal of clinical oncology: official journal of the American Society of Clinical Oncology. 2012;30(15):1796-804. 
17. Liedtke C, Mazouni C, Hess KR, Andre F, Tordai A, Mejia JA, Symmans WF, Gonzalez-Angulo AM, Hennessy B, Green M, et al. Response to neoadjuvant therapy and long-term survival in patients with triple-negative breast cancer. Journal of clinical oncology: official journal of the American Society of Clinical Oncology. 2008;26(8):1275-81.

18. Vargo JA, Beriwal S, Ahrendt GM, Soran A, Johnson RR, McGuire K, Bhargava R. Molecular class as a predictor of locoregional and distant recurrence in the neoadjuvant setting for breast cancer. Oncology. 2011;80(5-6):341-9.

19. Wang S, Yang H, Tong F, Zhang J, Yang D, Liu H, Cao Y, Liu P, Zhou P, Cheng L, et al. Response to neoadjuvant therapy and disease free survival in patients with triple-negative breast cancer. Gan to kagaku ryoho Cancer chemotherapy. 2009;36(2):255-8.

20. Carey LA, Dees EC, Sawyer L, Gatti L, Moore DT, Collichio F, Ollila DW, Sartor Cl, Graham ML, Perou $\mathrm{CM}$. The triple negative paradox: primary tumor chemosensitivity of breast cancer subtypes. Clinical cancer research: an official journal of the American Association for Cancer Research. 2007;13(8):2329-34.

21. Sikov WM, Berry DA, Perou CM, Singh B, Cirrincione CT, Tolaney SM, Somlo G, Port ER, Qamar R, Sturtz $K$, et al: Event-free and overall survival following neoadjuvant weekly paclitaxel and dosedense $A C+$ /- carboplatin and/or bevacizumab in triple-negative breast cancer. Outcomes from CALGB 40603 (Alliance). In: San Antonio Breast Cancer Symposium. San Antonio; 2015.

22. Geiger S, Cnossen JA, Horster S, DiGioia D, Heinemann V, Stemmler HJ: Long-term follow-up of patients with metastatic breast cancer. results of a retrospective, single-center analysis from 2000 to 2005. Anti-cancer drugs 2011, 22(9):933-939.

23. Kassam F, Enright K, Dent R, Dranitsaris G, Myers J, Flynn C, Fralick M, Kumar R, Clemons M. Survival outcomes for patients with metastatic triple-negative breast cancer: implications for clinical practice and trial design. Clin Breast Cancer. 2009;9(1):29-33.

24. Schmid P, Adams S, Rugo HS, Schneeweiss A, Barrios CH, Iwata H, Dieras V, Hegg R, Im SA, Shaw Wright G, et al. Atezolizumab and Nab-Paclitaxel in Advanced Triple-Negative Breast Cancer. N Engl J Med. 2018;379(22):2108-21.

25. Salgado R, Denkert C, Demaria S, Sirtaine N, Klauschen F, Pruneri G, Wienert S, Van den Eynden G, Baehner FL, Penault-Llorca F, et al. The evaluation of tumor-infiltrating lymphocytes (TILs) in breast cancer: recommendations by an International TILs Working Group 2014. Ann Oncol. 2015;26(2):259-71.

26. Voss K, Van der Auwera G, Gentry J: Full-stack genomics pipelining with GATK4 + WDL + Cromwell. In: 18th Annual Bioinformatics Open Source Conference: 20172017; Prague, Czech Republic, 2017.

27. Griffith M, Griffith OL, Smith SM, Ramu A, Callaway MB, Brummett AM, Kiwala MJ, Coffman AC, Regier AA, Oberkfell BJ, et al. Genome Modeling System: A Knowledge Management Platform for Genomics. PLoS Comput Biol. 2015;11(7):e1004274.

28. Aligning sequence reads, clone sequences and assembly contigs with BWA-MEM [https://arxiv.org/abs/1303.3997]. 
29. McKenna A, Hanna M, Banks E, Sivachenko A, Cibulskis K, Kernytsky A, Garimella K, Altshuler D, Gabriel S, Daly M, et al. The Genome Analysis Toolkit: a MapReduce framework for analyzing nextgeneration DNA sequencing data. Genome Res. 2010;20(9):1297-303.

30. Li H, Handsaker B, Wysoker A, Fennell T, Ruan J, Homer N, Marth G, Abecasis G, Durbin R. Genome Project Data Processing S: The Sequence Alignment/Map format and SAMtools. Bioinformatics. 2009;25(16):2078-9.

31. do Valle IF, Giampieri E, Simonetti G, Padella A, Manfrini M, Ferrari A, Papayannidis C, Zironi I, Garonzi M, Bernardi S, et al. Optimized pipeline of MuTect and GATK tools to improve the detection of somatic single nucleotide polymorphisms in whole-exome sequencing data. BMC Bioinformatics. 2016;17(Suppl 12):341.

32. Saunders CT, Wong WS, Swamy S, Becq J, Murray LJ, Cheetham RK. Strelka: accurate somatic smallvariant calling from sequenced tumor-normal sample pairs. Bioinformatics. 2012;28(14):1811-7.

33. Koboldt DC, Larson DE, Wilson RK. Using VarScan 2 for Germline Variant Calling and Somatic Mutation Detection. Curr Protoc Bioinformatics. 2013;44:15 14 11-7.

34. Tan A, Abecasis GR, Kang HM. Unified representation of genetic variants. Bioinformatics. 2015;31(13):2202-4.

35. Karczewski K, Francioli L, Tiao G, Cummings B, Alföldi J, Wang Q, Collins R, Laricchia K, Ganna A, Birnbaum D, et al: Variation across 141,456 human exomes and genomes reveals the spectrum of loss-of-function intolerance across human protein-coding genes: Supplementary Information; 2019.

36. Barnell EK, Ronning P, Campbell KM, Krysiak K, Ainscough BJ, Sheta LM, Pema SP, Schmidt AD, Richters $\mathrm{M}$, Cotto KC, et al. Standard operating procedure for somatic variant refinement of sequencing data with paired tumor and normal samples. Genet Med. 2019;21(4):972-81.

37. McLaren W, Gil L, Hunt SE, Riat HS, Ritchie GR, Thormann A, Flicek P, Cunningham F. The Ensembl Variant Effect Predictor. Genome Biol. 2016;17(1):122.

38. Yates AD, Achuthan P, Akanni W, Allen J, Allen J, Alvarez-Jarreta J, Amode MR, Armean IM, Azov AG, Bennett R, et al. Ensembl 2020. Nucleic Acids Res. 2020;48(D1):D682-8.

39. Liao Y, Wang J, Jaehnig EJ, Shi Z, Zhang B. WebGestalt 2019: gene set analysis toolkit with revamped Uls and APIs. Nucleic Acids Res. 2019;47(W1):W199-w205.

40. Damotte D, Warren S, Arrondeau J, Boudou-Rouquette P, Mansuet-Lupo A, Biton J, Ouakrim H, Alifano $M$, Gervais C, Bellesoeur A, et al. The tumor inflammation signature (TIS) is associated with anti-PD-1 treatment benefit in the CERTIM pan-cancer cohort. J TransI Med. 2019;17(1):357.

41. Vadakekolathu J, Minden MD, Hood T, Church SE, Reeder S, Altmann H, Sullivan AH, Viboch EJ, Patel $\mathrm{T}$, Ibrahimova $\mathrm{N}$, et al: Immune landscapes predict chemotherapy resistance and immunotherapy response in acute myeloid leukemia. Sci Transl Med 2020, 12(546).

42. Sharma P, Kimler BF, O'Dea A, Nye EL, Wang YY, Yoder R, Prochaska LH, Wagner JL, Amin AL, Larson $\mathrm{K}$, et al: Results of randomized phase II trial of neoadjuvant carboplatin plus docetaxel or carboplatin plus paclitaxel followed by AC in stage I-III triple-negative breast cancer (NCT02413320). In: American Society of Clinical Oncology: 2019; Chicago, IL.; 2019. 
43. Loibl S, Weber KE, Timms KM, Elkin EP, Hahnen E, Fasching PA, Lederer B, Denkert C, Schneeweiss A, Braun S, et al. Survival analysis of carboplatin added to an anthracycline/taxane-based neoadjuvant chemotherapy and HRD score as predictor of response-final results from GeparSixto. Ann Oncol. 2018;29(12):2341-7.

44. Hahnen E, Lederer B, Hauke J, Loibl S, Krober S, Schneeweiss A, Denkert C, Fasching PA, Blohmer JU, Jackisch C, et al. Germline Mutation Status, Pathological Complete Response, and Disease-Free Survival in Triple-Negative Breast Cancer: Secondary Analysis of the GeparSixto Randomized Clinical Trial. JAMA Oncol. 2017;3(10):1378-85.

45. Sikov WM, Polley M, Twohy E, Perou CM, Singh B, Berry DA, Tolaney SM, Somlo G, Port ER, Ma CX, et al: CALGB (Alliance) 40603: Long-term outcomes (LTOs) after neoadjuvant chemotherapy (NACT) +/carboplatin (Cb) and bevacizumab (Bev) in triple-negative breast cancer (TNBC). In: American Society of Clinical Oncology Annual Meeting 2019: 2019; Chicago, IL; 2019.

46. Doxorubicin Hydrochloride and Cyclophosphamide Followed by Paclitaxel With or Without Carboplatin in Treating Patients With Triple-Negative Breast Cancer NCT02488967 https://clinicaltrials.gov/ct2/show/NCT02488967.

47. Adjuvant Treatment of EC Followed by Taxane +/- Carboplatin in Triple-Negative Breast Cancer (TCTN) NCT02455141 https://clinicaltrials.gov/ct2/show/NCT02455141.

48. Carboplatin in EARLY Triple Negative Breast Cancer Trial (PEARLY Trial) NCT02441933 [https://clinicaltrials.gov/ct2/show/NCT02441933].

49. Cortazar P, Geyer CE Jr. Pathological complete response in neoadjuvant treatment of breast cancer. Ann Surg Oncol. 2015;22(5):1441-6.

50. Cortazar P, Zhang L, Untch M, Mehta K, Costantino JP, Wolmark N, Bonnefoi H, Cameron D, Gianni L, Valagussa $\mathrm{P}$, et al. Pathological complete response and long-term clinical benefit in breast cancer: the CTNeoBC pooled analysis. Lancet. 2014;384(9938):164-72.

51. Symmans WF, Wei C, Gould R, Yu X, Zhang Y, Liu M, Walls A, Bousamra A, Ramineni M, Sinn B, et al. Long-Term Prognostic Risk After Neoadjuvant Chemotherapy Associated With Residual Cancer Burden and Breast Cancer Subtype. Journal of clinical oncology: official journal of the American Society of Clinical Oncology. 2017;35(10):1049-60.

52. Tung N, Arun B, Hacker MR, Hofstatter E, Toppmeyer DL, Isakoff SJ, Borges V, Legare RD, Isaacs C, Wolff AC, et al. TBCRC 031: Randomized Phase II Study of Neoadjuvant Cisplatin Versus Doxorubicin-Cyclophosphamide in Germline BRCA Carriers With HER2-Negative Breast Cancer (the INFORM trial). Journal of clinical oncology: official journal of the American Society of Clinical Oncology. 2020;38(14):1539-48.

53. Loibl S, O'Shaughnessy J, Untch M, Sikov WM, Rugo HS, McKee MD, Huober J, Golshan M, von Minckwitz G, Maag D, et al. Addition of the PARP inhibitor veliparib plus carboplatin or carboplatin alone to standard neoadjuvant chemotherapy in triple-negative breast cancer (BrighTNess): a randomised, phase 3 trial. Lancet Oncol. 2018;19(4):497-509. 
54. Gluz O, Nitz U, Liedtke C, Christgen M, Grischke EM, Forstbauer H, Braun M, Warm M, Hackmann J, Uleer C, et al. Comparison of Neoadjuvant Nab-Paclitaxel + Carboplatin vs Nab-Paclitaxel + Gemcitabine in Triple-Negative Breast Cancer: Randomized WSG-ADAPT-TN Trial Results. J Natl Cancer Inst. 2018;110(6):628-37.

55. Groheux D, Biard L, Lehmann-Che J, Teixeira L, Bouhidel FA, Poirot B, Bertheau P, Merlet P, Espie M, Resche-Rigon $\mathrm{M}$, et al. Tumor metabolism assessed by FDG-PET/CT and tumor proliferation assessed by genomic grade index to predict response to neoadjuvant chemotherapy in triple negative breast cancer. Eur J Nucl Med Mol Imaging. 2018;45(8):1279-88.

56. Riva F, Bidard FC, Houy A, Saliou A, Madic J, Rampanou A, Hego C, Milder M, Cottu P, Sablin MP, et al. Patient-Specific Circulating Tumor DNA Detection during Neoadjuvant Chemotherapy in TripleNegative Breast Cancer. Clin Chem. 2017;63(3):691-9.

57. Chen YH, Hancock BA, Solzak JP, Brinza D, Scafe C, Miller KD, Radovich M. Next-generation sequencing of circulating tumor DNA to predict recurrence in triple-negative breast cancer patients with residual disease after neoadjuvant chemotherapy. NPJ Breast Cancer. 2017;3:24.

58. Teng YH, Tan WJ, Thike AA, Cheok PY, Tse GM, Wong NS, Yip GW, Bay BH, Tan PH. Mutations in the epidermal growth factor receptor (EGFR) gene in triple negative breast cancer: possible implications for targeted therapy. Breast Cancer Res. 2011;13(2):R35.

59. Cancer Genome Atlas N. Comprehensive molecular portraits of human breast tumours. Nature. 2012;490(7418):61-70.

60. Lee SW, Choi D, Heo M, Shin EC, Park SH, Kim SJ, Oh YK, Lee BH, Yang SH, Sung YC, et al. hIL-7-hyFc, A Long-Acting IL-7, Increased Absolute Lymphocyte Count in Healthy Subjects. Clin Transl Sci. 2020;13(6):1161-9.

61. Kim JH, Kim YM, Choi D, Jo SB, Park HW, Hong SW, Park S, Kim S, Moon S, You G, et al. Hybrid Fcfused interleukin-7 induces an inflamed tumor microenvironment and improves the efficacy of cancer immunotherapy. Clin Transl Immunology. 2020;9(9):e1168.

\section{Figures}




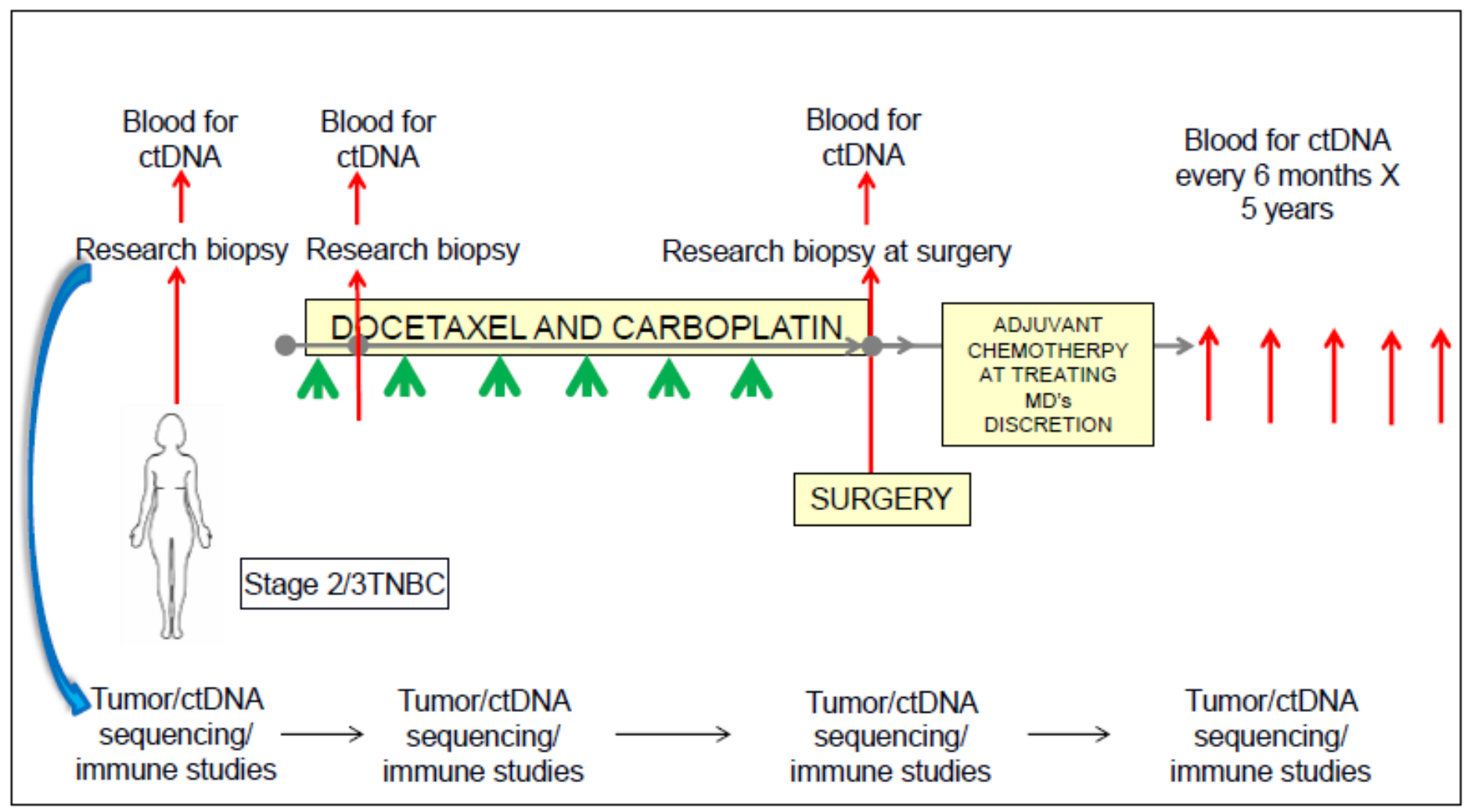

Figure 1

Clinical trial schema. Diagram of clinical trial showing chemotherapy regimen and biospecimen collection time points. 


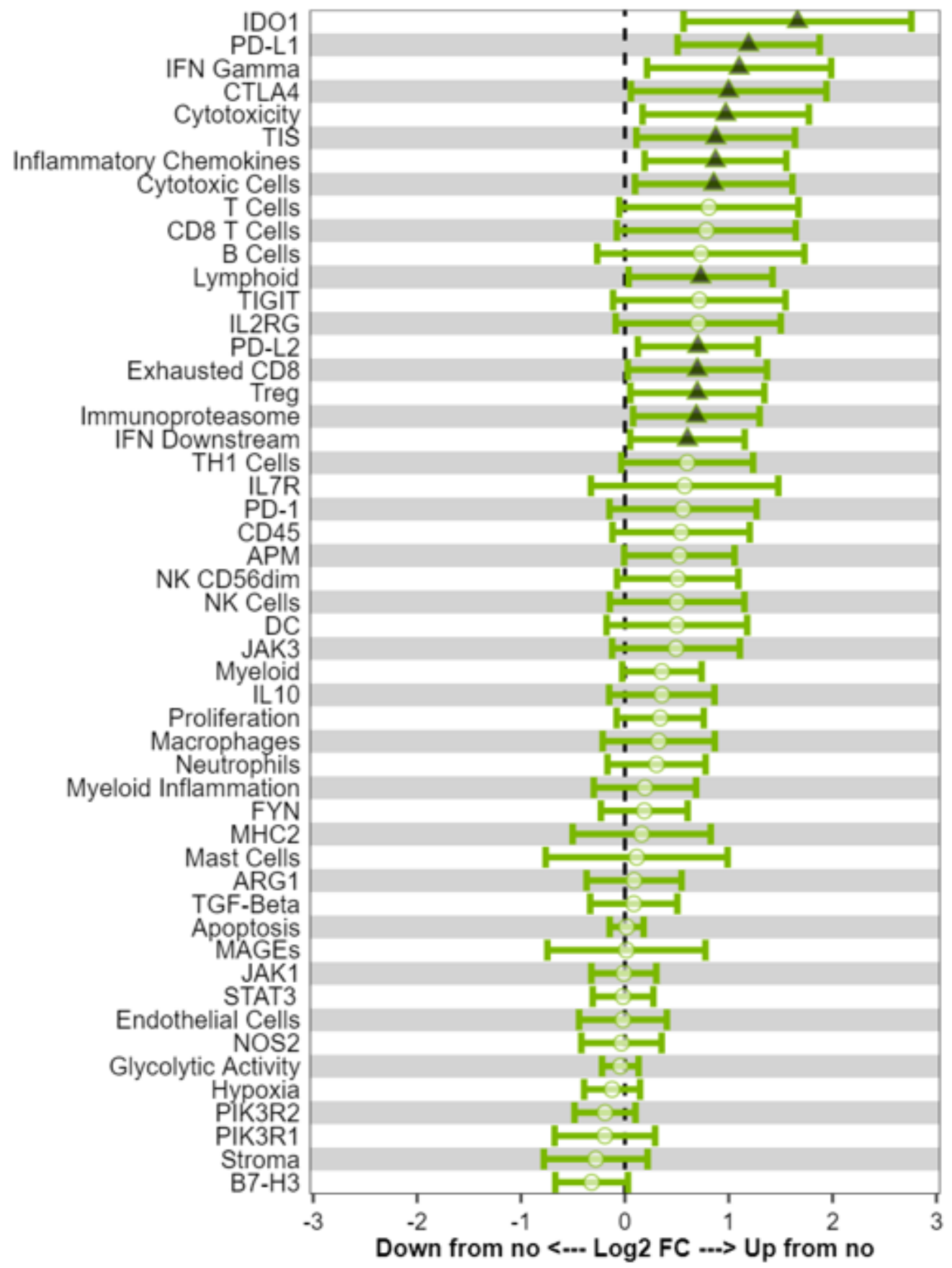

Significance $\nabla$ Down $\bigcirc$ Not $\mathbf{A}$ Up

\section{Figure 2}

Differential expression of gene signatures' forest plot based on PCR in baseline samples only. This shows the differential expression means and $95 \%$ confidence intervals between response variables, for each signature on an unadjusted scale. The vertical axis is shown at fold change equal to zero, indicating equivalent expression between pCR and no pCR (no). As the marker shifts from the center line there is an increase (shift to the right), or decrease (shift to the left), in the differential expression of that signature when compared to the no pCR group (represented as the vertical line at zero). The shape of the marker in 
each box indicates whether there is a significant difference in the signature as assessed by univariate analysis (note that this significance is not adjusted for multiple comparisons). A signature is considered significant if the $95 \%$ confidence interval (the horizontal line of the signature) does not cross the vertical axis representing the no response group.

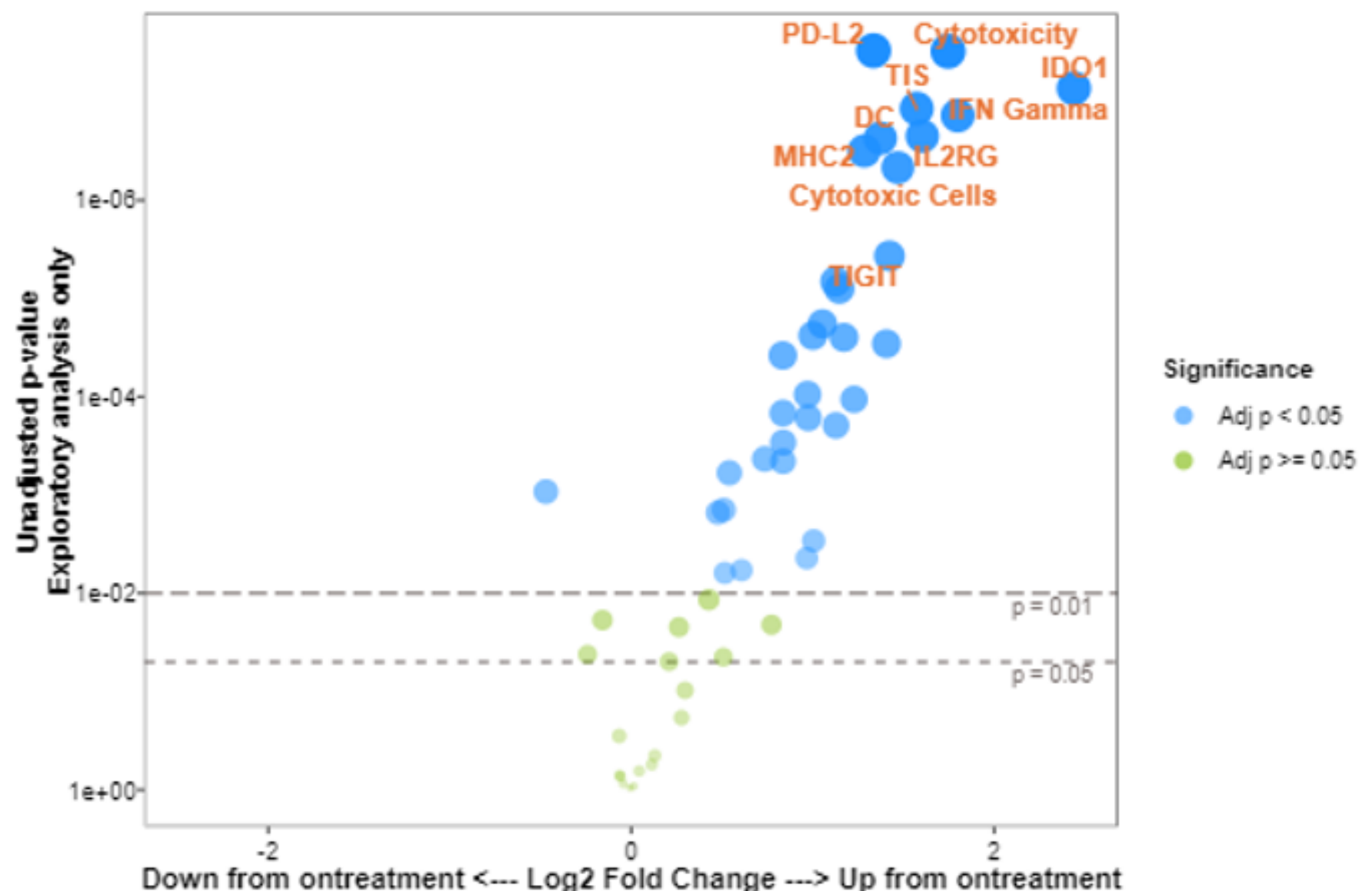

Figure 3

Differential expression of 10360 gene signatures before and on-treatment. A) Volcano plot showing differential expression of 10360 immune signatures from baseline or on treatment samples. Signatures in blue were significantly different using an adjusted $p$-value $(p<0.05)$. Dotted lines indicate an unadjusted $p$-value of $p<0.05$ and $p<0.01$. Larger circles indicate greater significance. 


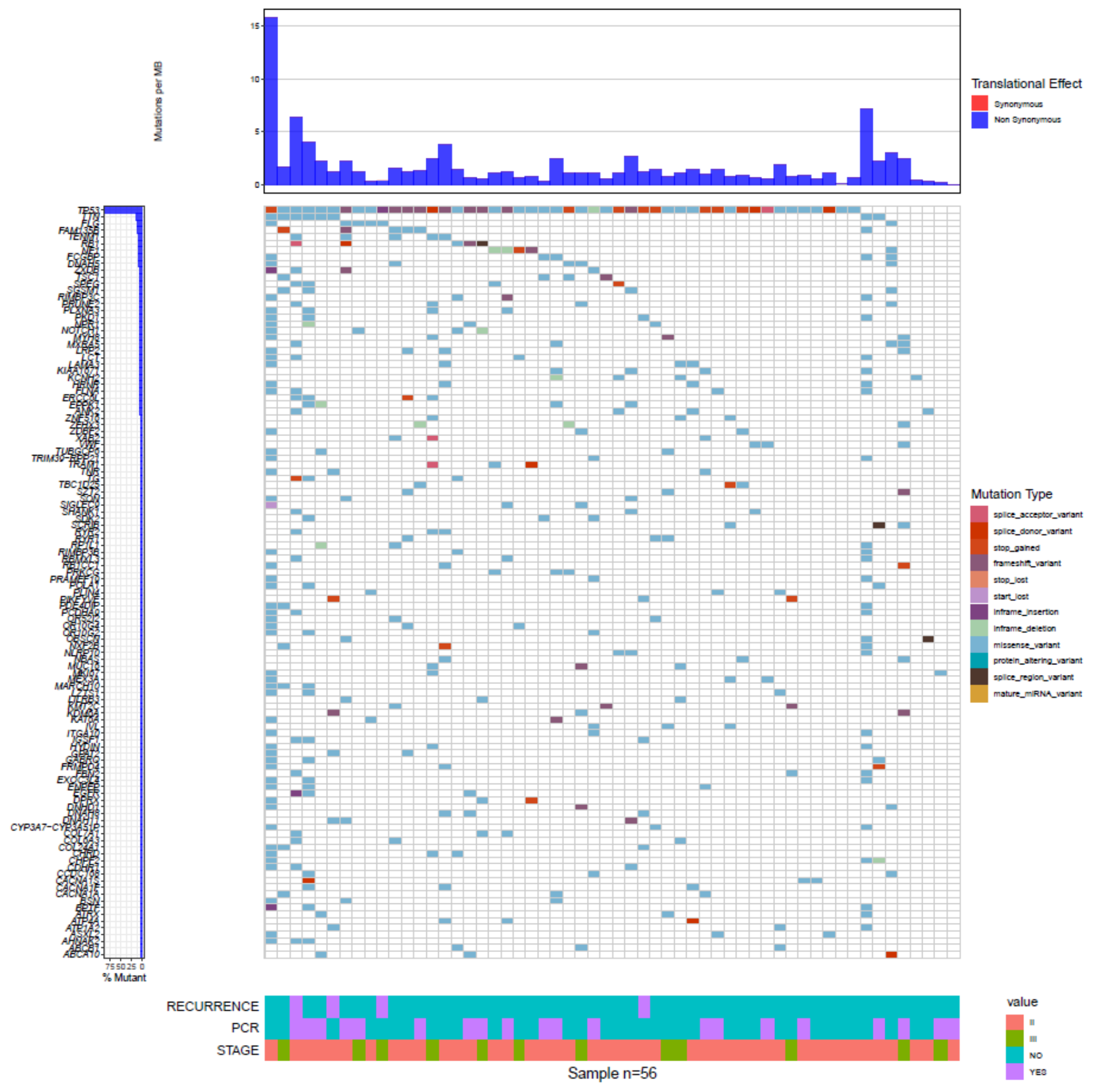

\section{Figure 4}

The mutation landscape waterfall plot of genes with a non-synonymous (frameshift, in-frame deletion, inframe insertion, mature miRNA, missense, protein altering, splice acceptor, splice donor, splice region, start lost, stop gained, and stop lost) mutation frequency $>5 \%$. Top bar plot indicates mutation burden, left bar plot indicates mutation frequency, and lower panel provides clinical annotation. 


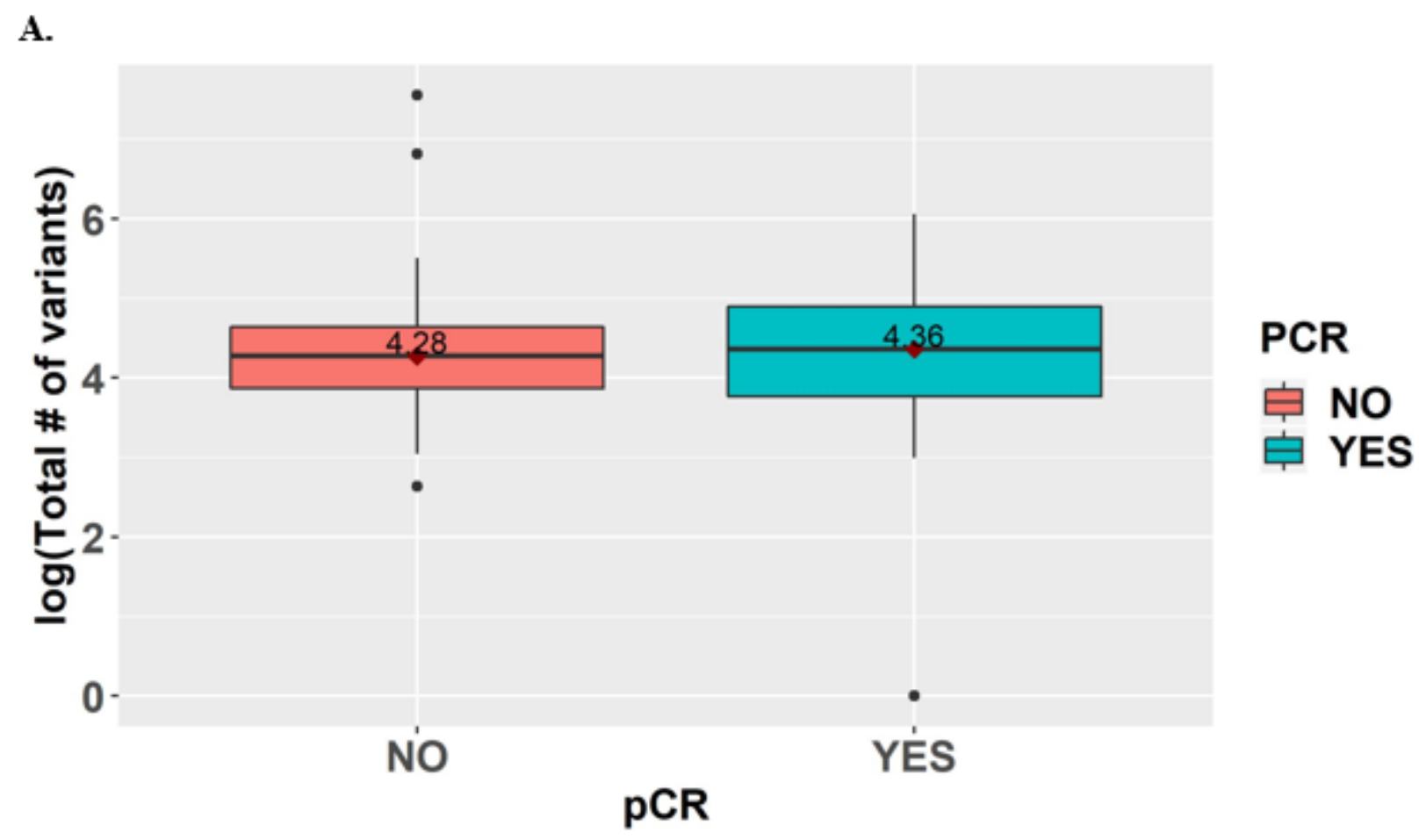

B.

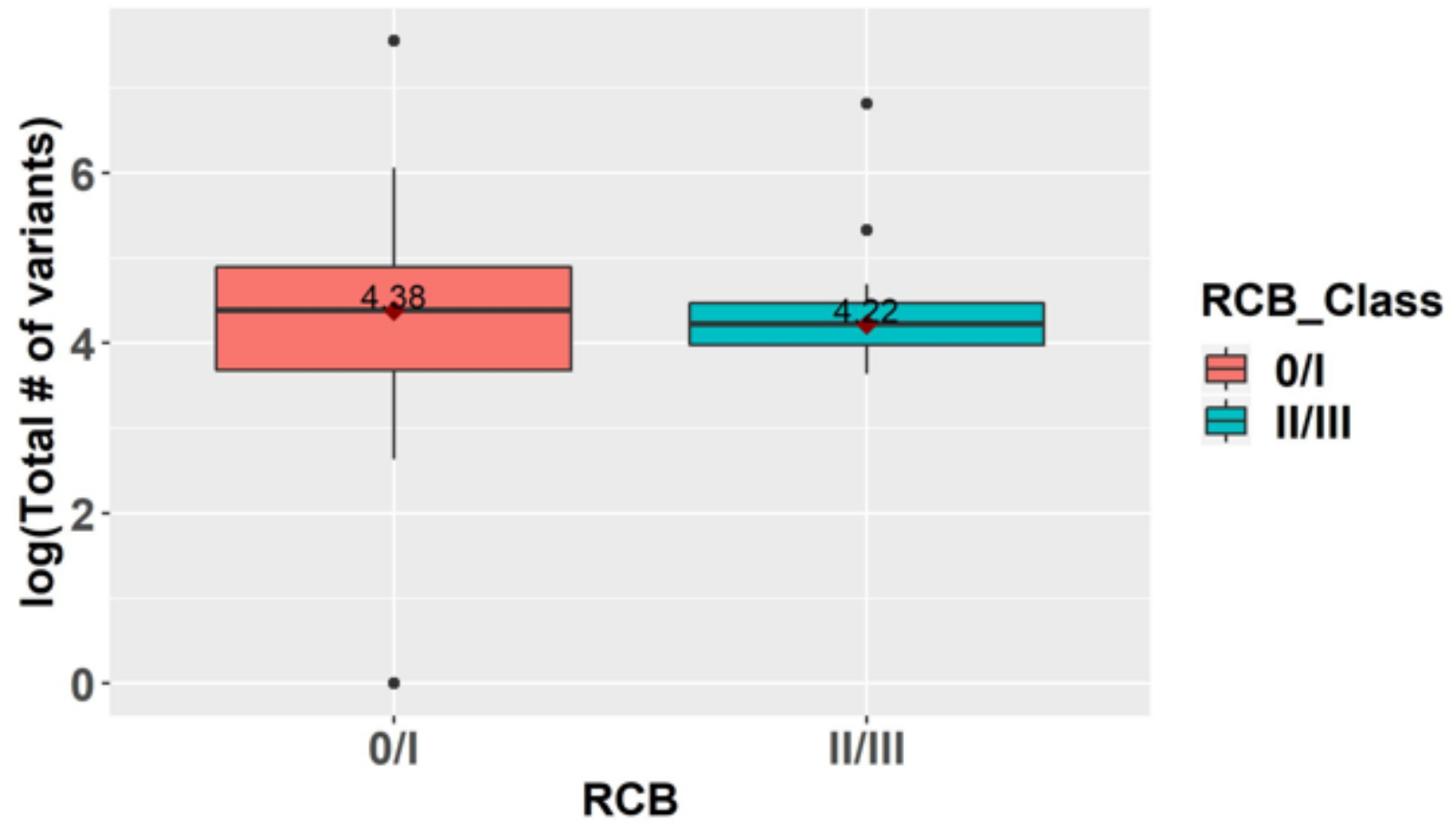

Figure 5

Overall mutation burden according to pathological response. Log scale of somatic mutation burden according to pathological response following neoadjuvant chemotherapy. Red bar indicates patients without pCR. Green bar indicates patients with pCR. pCR, pathological complete response. 

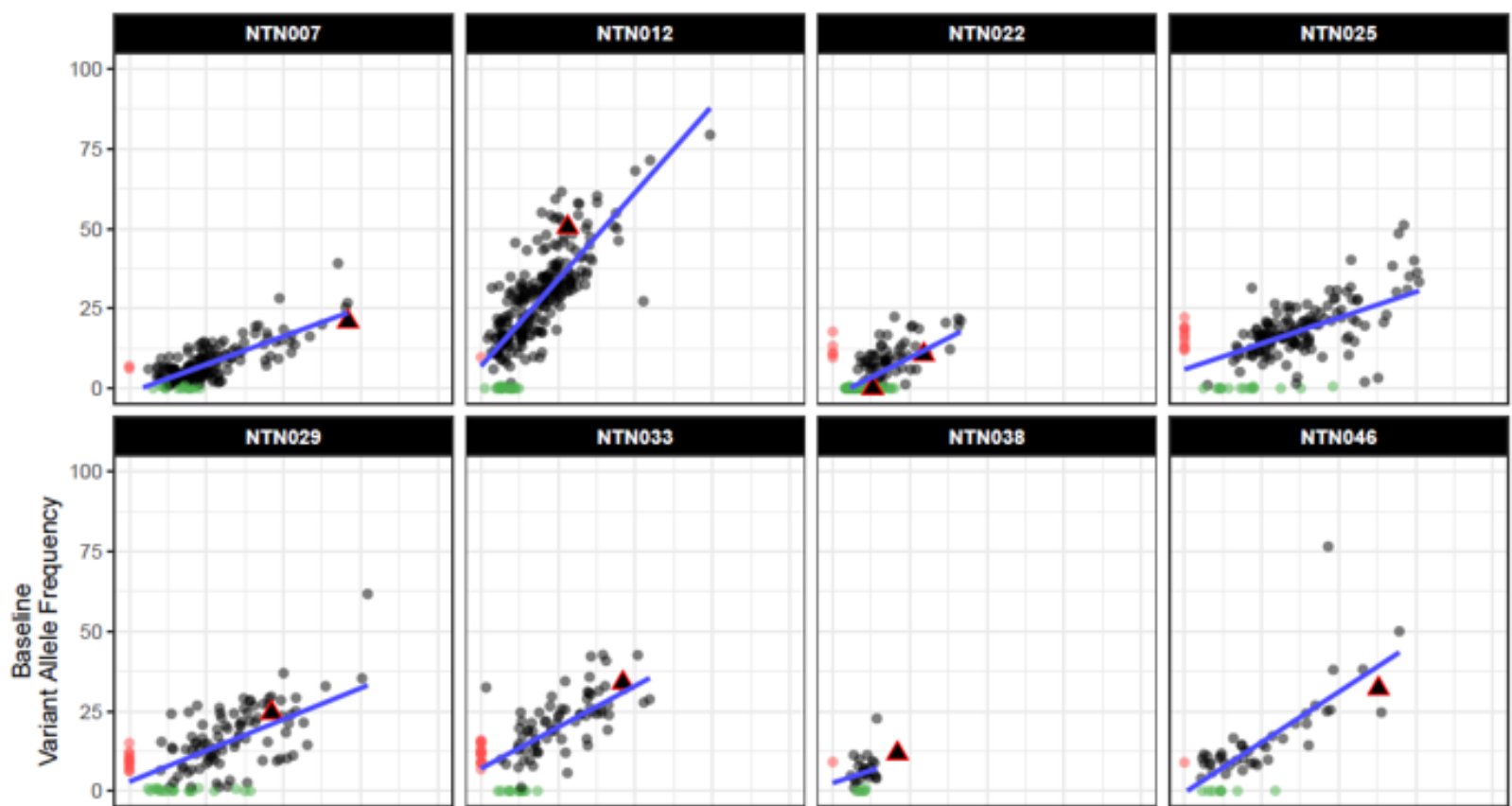

Clonal Status

- Shared/Clonal

- Subcional Depleted

Subcional Selected
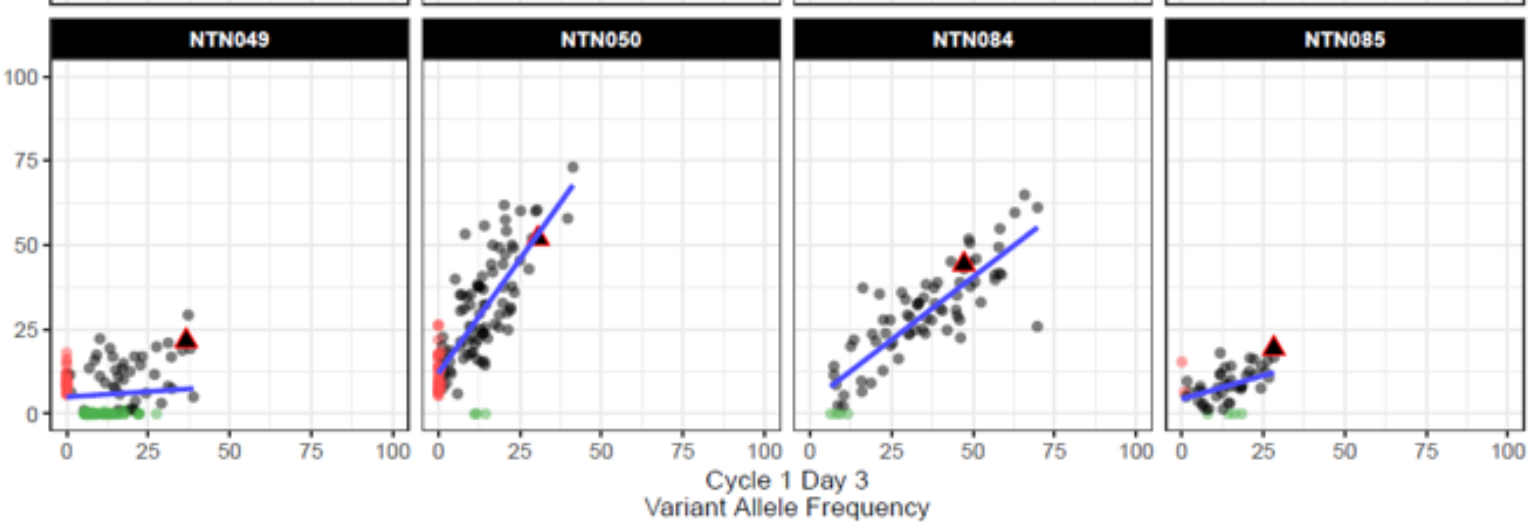

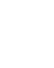

Figure 6

Comparison of variant detection in pre-treatment and on-treatment paired tumor samples. Of the 13 participants for which WES was performed at both baseline and C1D3, 12 matched samples are plotted. One paired sample set is not shown due to low variant counts. For the variants plotted, the minimum coverage was set to 30 reads. The clonal status of variants in each sample is indicated by color. Red represents subclonal depleted mutations, green represents subclonal selected mutations, and grey represents shared clonal mutations. TP53 variants were called in 11 of the participants' samples and are highlighted in the plots by the triangle.

\section{Supplementary Files}

This is a list of supplementary files associated with this preprint. Click to download.

- Additionalfile1.docx

- Additionalfile2.docx 
- Additionalfile3.docx

- Additionalfile4.docx

- Additionalfile5.docx

- Additionalfile6.docx 